\title{
Similarities among ectoparasite fauna of sigmodontine rodents: phylogenetic and geographical influences
}

\author{
LEONARDO DOMINICI CRUZ ${ }^{1} \dagger^{*}$, FERNANDA RODRIGUES FERNANDES ${ }^{2} \dagger$ \\ and ARÍCIO XAVIER LINHARES ${ }^{3}$ \\ ${ }^{1}$ Programa de Pós-graduação em Ciências Biológicas (Zoologia), Instituto de Biociências, Universidade Estadual Paulista \\ fúlio de Mesquita Filho, Rio Claro, São Paulo, Brazil \\ ${ }_{2}^{2}$ Programa de Pós-graduação em Ecologia, Instituo de Biologia, Universidade Estadual de Campinas, Campinas, São Paulo, \\ Brazil \\ ${ }^{3}$ Departamento de Biologia Animal, Instituto de Biologia, Universidade Estadual de Campinas, Campinas, São Paulo, \\ Brazil
}

(Received 11 February 2012; revised 6 April, 23 May and 27 May 2012; accepted 27 May 2012; first published online 20 August 2012)

\section{S UMMARY}

Phylogenetic and geographical overlaps in host distributions influence the compositional similarity of ectoparasite fauna in a host-parasite system. In these systems, hosts that are more closely related (phylogenetically) are expected to share more parasitic species than more distantly related hosts. Similarly, hosts sharing a larger geographical distribution overlap are expected to have similar ectoparasites. This study investigated the influence of phylogeny (divergence time) and geographical overlap of some neotropical sigmodontine rodent species on the similarities among their ectoparasite fauna (Mesostigmata and Siphonaptera), using a partial Mantel test. Divergence time was the only significant factor that influenced the similarity among the ectoparasites, when mites and fleas were analysed together. Host species that had diverged more recently displayed ectoparasite fauna that were similar. The similarities of the flea species showed similar results in both separate and joint analyses, but neither phylogenetic nor geographical overlap influenced the similarity in mite species. Fleas were shown to be more host-specific than were mesostigmate mites, probably because of the increased influence of host phylogeny.

Key words: flea, geographical overlap, mammal, mite, phylogeny.

\section{INTRODUCTION}

Parasite fauna is the full range of parasite species that exploits a host species throughout its geographical range (Poulin, 2007). The range of parasites that are associated with a particular host species may be influenced by evolution and is primarily influenced by the acquisition and loss of parasite species over time. A host species acquires a parasitic species either by inheritance from ancestral host species (Brooks, 1988) or by exchange with other related or unrelated host species that co-occur in the same geographical area (Antonovics et al. 2002; Brooks et al. 2006). In contrast, the loss of an associated parasite species can occur through historical 'accidents' (e.g., the abrupt division of a host population in which a founding population becomes isolated and free of a specific parasite species) or by the dispersal of the host species to new environments, which present unfavourable

* Corresponding author: Universidade Federal do Maranhão, Campus de São Bernardo, Rua Projetada, s/n, Perímetro Urbano, 65550000, São Bernardo, Maranhão, Brazil. Tel: +559834771513. E-mail: leonardo. dominici@gmail.com

† Current address: Universidade Federal do Maranhão, Campus de São Bernardo, São Bernardo, Maranhão, Brazil. conditions for some parasite species (e.g., the absence of an appropriate intermediate host in the new environment) (Hafner and Page, 1995; Paterson et al. 1999). Because of these processes, the composition of the parasite fauna for a particular host species is a result of both its phylogenetic identity and its environment (Kennedy and Bush, 1994). Accordingly, the phylogenetic effect is expected to manifest through a greater compositional similarity among the parasites of host species with closer phylogenetic relatedness. The effect of the environment may be manifest through greater compositional similarity among the parasite fauna associated with host species that co-occur within a certain environment (e.g., the same geographical area), regardless of their phylogenetic relationship (Hoberg and Brooks, 2008).

Recent studies have examined the influence of host phylogeny and environment on the composition of parasite communities in some parasite-host systems (e.g., Brooks et al. 2006; Muñoz et al. 2006; Davies and Pedersen, 2008; Poulin, 2010). In ectoparasiterodent systems, Krasnov et al. (2010) showed that both host phylogeny and environmental factors influence the compositional similarities among the flea and mite communities in the Palaearctic region. Krasnov et al. (2012) further showed that host 
phylogeny has a greater influence, than environmental factors, on the composition of flea communities parasitizing small mammals in the Neotropical region. Smith et al. (2008) showed that host shift was the major factor influencing the composition of lice communities in rodents that co-occur in the same area. Both Smith et al. (2008) and Krasnov et al. (2012) presented results for the Neotropical region, but the former group presented only data with lice, while the latter group presented data from 4 biogeographical regions, with only 8 out of the 63 published studies presenting data from the Neotropical region.

In South America, the Sigmodontinae subfamily of rodent species contribute the majority of the species richness amongst terrestrial mammals and includes the most common species in small mammal assemblages. Most studies indicate that the ancestors of the sigmodontine rodents originated in Central and/or North America, invading South America approximately 10-14 MYA. Once these rodents became established, they underwent a very rapid radiation, followed by differentiation into tribes and genera (Smith and Patton, 1999; Steppan et al.2004). In fact, sigmodontine rodents have a high diversity of forms and occupy a wide variety of niches, from cold montane woodlands to lowland rainforests, dry coastal deserts, and temperate grasslands with lifestyles ranging from semiaquatic to fossorial, arboreal, and scansorial. Although their evolutionary history and ecology are relatively well documented, studies about the influence of these factors on the compositional similarities of their ectoparasite fauna are scarce.

The factors that influence the composition of the ectoparasite fauna of rodents vary between biogeographical regions because each region presents a unique set of species and environmental characteristics, as well as differences in the levels of host specialization by the parasites and differences in the history of the host-parasite associations (Krasnov et al. 2012). Therefore, the aim of this study was to investigate the influence of the phylogeny (represented by divergence time) and environment (represented by overlap in geographical distribution) of some sigmodontine rodent species on the compositional similarity of their ectoparasites (mesostigmate mites and fleas). This study was designed to contribute to the understanding of the rodentparasite systems in the Neotropical region. The comparatively recent and very rapid radiation of sigmodontine rodents implies that their ectoparasites have had less evolutionary time to switch between distantly related hosts. In addition, because ectoparasites can also parasitize other host species that co-occur in the same geographical area, our hypothesis was that both phylogeny and geographical overlap influenced the compositional similarity of arthropod ectoparasite fauna parasitizing different sigmodontine rodent species.
MATERIALS AND METHODS

\section{Arthropod ectoparasite data}

Studies on ectoparasites (fleas and mites) of sigmodontine rodents are scarce in the neotropics and most of the published studies were conducted in the southeastern and southern regions of Brazil. Thus, the data collected on ectoparasitic arthropods from Sigmodontinae rodents in the different regions of southeastern/southern Brazil were obtained from 15 published and unpublished works (Appendix Table A1; online version only) that recorded the species of mites (Acari: Mesostigmata) or fleas (Insecta: Siphonaptera) found on each rodent species. Some of these rodent species are also distributed across other South American countries; therefore, only a subset of their parasite fauna was considered in this study. Rodent species with less than 5 individual ectoparasites recorded were excluded from the analysis; unique individual records of mesostigmate mite or flea species on a host species were also excluded from the analysis. In total, data for 20 species of mesostigmate mites and 14 species of fleas collected from 16 or 15 rodent species, respectively, were used (in Appendix Tables A2 and A3; online version only).

\section{Ectoparasite fauna description}

The host ectoparasite fauna was determined by the mean number of ectoparasite species per host species. The host specificity of each mesostigmate mite and flea species was determined by the average taxonomic distinctness index, $S_{\mathrm{TD}}$, because this index incorporates the phylogenetic relationship amongst the host species (Poulin and Mouillot, 2003):

$S_{T D}=2 \frac{\sum \sum_{i<j} \omega_{i j}}{s(s-1)}$

where $s$ is the number of host species used by an ectoparasite and $\omega_{i j}$ is the taxonomic distinctness between host species $i$ and $j$. In this study, the $S_{\mathrm{TD}}$ index was based on a 4-step taxonomic hierarchy (species, genus, tribe, and subfamily) and was limited to mesostigmate mites and flea species recorded in more than 1 rodent host species. Thus, mesostigmate mite and flea species with $S_{\mathrm{TD}}$ values close to 3 show lower host specificity than those with a $S_{\mathrm{TD}}$ value close to 1 , which show high host specificity.

\section{Compositional similarity matrix of ectoparasite faunas}

A matrix of the presence/absence of each arthropod ectoparasite species was generated. In this matrix, the presence of an ectoparasite species on a rodent host species was represented by 1 , whereas its absence was represented by 0 . The compositional similarity of the ectoparasite faunas between the rodent host species 
was calculated using Jaccard's index $\left(\beta_{j}\right)$ because this is one of the most frequently used indices in the literature and the statistical properties are well known (Krebs, 1999; Koleff et al. 2003):

$\beta_{j}=a /(a+b+c)$

where $a$ is the number of ectoparasite species present in 2 rodent host species, $X$ and $Y$; $b$ is the number of ectoparasite species present on host species $X$ and absent on host species $Y$; and $c$ is the number of ectoparasite species present on host species $Y$ and absent on host species $X$. The similarities among the rodent species were first calculated including all of the ectoparasite taxa and then were calculated separately for each ectoparasite taxon (mesostigmate mites and fleas). This procedure was performed using the environment 2.12.2 (R Development Core Team, 2010) vegan package v.1.17-10 (Okasen et al. 2011).

\section{Estimates of the divergence times of host species}

Estimates of the divergence times for the rodent host species were obtained through the construction of a phylogeny tree for these species, based on evolutionary models of nucleotide base replacement. To this end, the mitochondrial cytochrome b gene sequences $(c y t b)$, obtained from GenBank (http://www.ncbi. nlm.nih.gov/genbank) (in Appendix Table A4; online version only), were used. These sequences were imported using BioEdit v 7.0.9 and aligned using the accessory program ClustalW (Hall, 2007). The program jModel'Test was used to select the bestfit model of nucleotide substitution by the Bayesian information criterion (BIC) (Posada, 2008). The 3-parameter model (Kimura, 1981), including a proportion of invariant sites and rate variation among sites (the model TPM3 uf $+\mathrm{I}+\mathrm{G}$ ), was the model selected $(\mathrm{BIC}=19855 \cdot 79, \Delta=0)$. The phylogenetic analysis was performed using Bayesian inference with the BEAST program $\mathrm{v}$ 1.5.4 (Drummond and Rambaut, 2007) and to assess statistical support for hypothesized clades, posterior distributions were obtained by the Markov chain Monte Carlo method (MCMC), with samples of trees, and parameters were drawn every 1000 steps from a total of 40 million MCMC steps. Because rodent species differ in rates of $c y t b$ evolution (Spradling et al. 2001), the host phylogeny was built under a relaxed clock model with branchspecific rates following a lognormal distribution (Drummond et al. 2006). The divergence time between hosts species was estimated based on 2 independent fossil calibrations, namely, the NecromysAkodon split (4 MYA) (Reig, 1987) and the earliest fossil record of Oligoryzomys flavescens in South American (1.5 MYA) (Pardiñas et al. 2002). The host phylogenetic tree obtained in this study resembles other proposed sigmodontine phylogenetic trees (e.g., D’Elía, 2003; Weksler, 2003). The few topological differences observed among our phylogeny and these early sigmodontine phylogenies may be the result of the smaller number of species used in this study for the construction of the host phylogenetic tree. Nevertheless, the determined host phylogeny is robust with most clades having posterior probabilities greater than $0 \cdot 8$ (in Appendix Fig. A1; online version only).

\section{Geographical overlap}

Overlapping geographical distributions of host species were calculated using a presence/absence matrix. A spatial resolution grid of $1^{\circ}$ latitude $\times 1^{\circ}$ longitude was constructed over a map of South America. Using the overlap of digital maps of the geographical distributions of rodent host species (Patterson et al. 2007), a presence/absence matrix was built, with 1 indicating the presence of the species in each cell of the map and 0 indicating the absence. The geographical overlap between the distributions of the host species was quantified using Jaccard's index $\left(\beta_{j}\right)$. This procedure was performed using the SAM software v 4.0 (Rangel et al. 2010).

\section{Data analysis}

A partial Mantel test was used to analyse the influence of the phylogeny (divergence time) and geography (geographical overlap) of the sigmodontine rodent species on the similarity of their arthropod ectoparasite faunas (Smouse et al. 1986). The partial Mantel test is a non-parametric correlation matrix, which accounts for the lack of independence of among the elements of each matrix and computes a statistic that is related to multiple regression coefficients (Smouse et al. 1986). To test the null hypothesis of no correlation, a procedure of randomization by the Monte Carlo method was employed to produce a null distribution. The probability of the null hypothesis was estimated directly by counting the number of randomizations in which the statistical test was less than or equal to the value obtained from the original matrix and dividing this number by the randomization number (10000 permutations) (Manly, 1991). The partial Mantel test was performed 3 times: the first test was performed considering the compositional similarities of the ectoparasite faunas in general (Mesostigmata and Siphonaptera); the second considering the compositional similarities of the mite faunas (Mesostigmata), and the third considering the compositional similarities of the flea faunas (Siphonaptera). This procedure was performed using the PASSaGE $\mathrm{v} \quad 2.0$ software (Rosenberg and Anderson, 2011).

\section{RESULTS}

In general, when the Mesostigmata and Siphonaptera species were combined, the mean size of the 


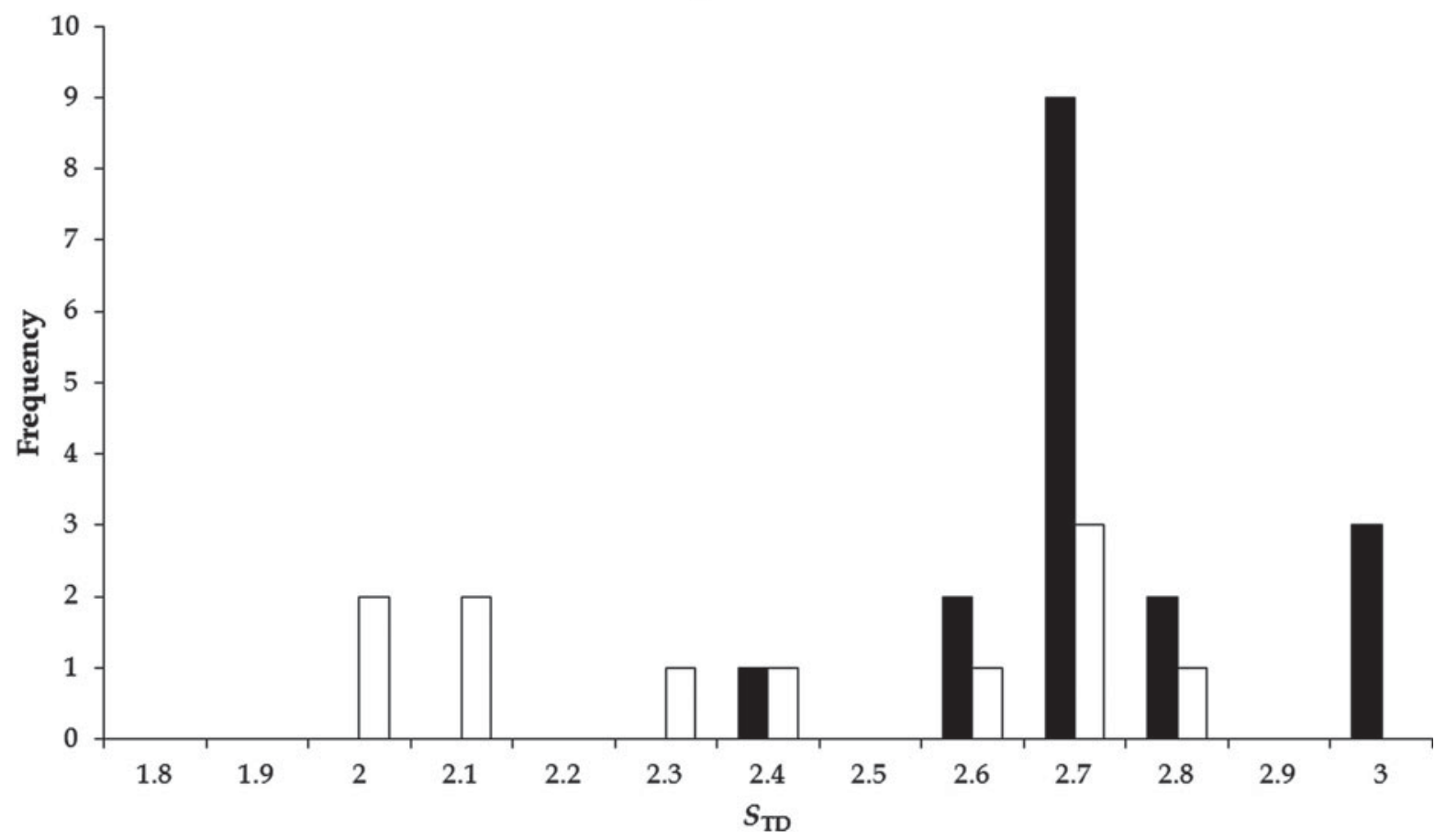

Fig. 1. Frequency distribution of $S_{\mathrm{TD}}$ values among mesostigmate mite and flea species parasitic on sigmodontine rodents.

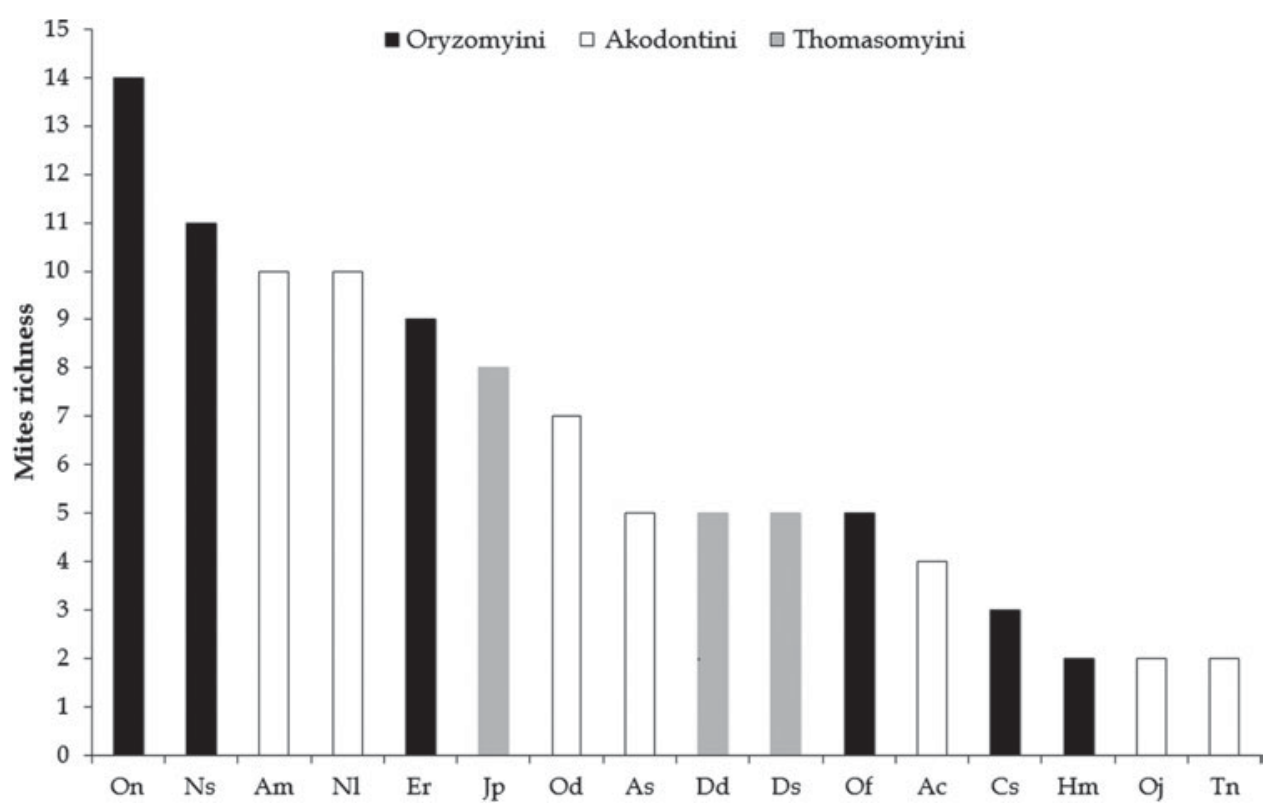

Fig. 2. The distribution of mite species richness on rodent hosts. (Hosts: On, Oligoryzomys nigripes; Ns, Nectomys squamipes; Am, Akodon montensis; Nl, Necromys lasiurus; Er, Euryoryzomys russatus; Fp, Fuliomys pictipes; Od, Oxymycterus dasytrichus; As, Akodon serrensis; Dd, Delomys dorsalis; Ds, Delomys sublineatus; Of, Oligoryzomys flavescens; Ac, Akodon cursor; Cs, Cerradomys suflavus; Hm, Hylaeamys megacephalus; Oj, Oxymycterus judex; and Tn, Thaptomys nigrita).

ectoparasite faunas was $9 \cdot 94$ species of ectoparasites per rodent host species, with $11 \cdot 7 \%$ of the pairs of rodent species sharing at least 1 species of ectoparasite. The greatest similarity in ectoparasite fauna among the rodent species was between Akodon montensis and Nectomys squamipes $\left(\beta_{j}=0.68\right)$.
Examination of only the Mesostigmata parasites, there were an average of 6.37 mesostigmate mite species per rodent host species, with $21 \cdot 7 \%$ of the pairs of host species sharing at least 1 species of mesostigmate mite. The greatest mesostigmate mite species similarity among the rodent species was also 
Table 1. Summary of the partial Mantel test results of shared ectoparasites $\left(\beta_{j}\right)$ versus the divergence time and geographical overlap for each subset of ectoparasite

\begin{tabular}{|c|c|c|c|c|}
\hline Ectoparasite & Independent variable & $R$ & $z$ & $P$ \\
\hline \multirow[t]{2}{*}{ Total } & Divergence time & $-0 \cdot 162$ & $-15 \cdot 154$ & $0 \cdot 017$ \\
\hline & Geographical overlap & $0 \cdot 057$ & $0 \cdot 443$ & $0 \cdot 695$ \\
\hline \multirow[t]{2}{*}{$H_{0}$} & Divergence time & & & $0 \cdot 019$ \\
\hline & Geographical overlap & & & $0 \cdot 712$ \\
\hline \multirow[t]{2}{*}{ Mesostigmata } & Divergence time & $-0 \cdot 014$ & $-1 \cdot 204$ & $0 \cdot 839$ \\
\hline & Geographical overlap & $-0 \cdot 047$ & $-0 \cdot 289$ & $0 \cdot 761$ \\
\hline \multirow[t]{2}{*}{$H_{0}$} & Divergence time & & & $0 \cdot 836$ \\
\hline & Geographical overlap & & & $0 \cdot 775$ \\
\hline \multirow[t]{2}{*}{ Siphonaptera } & Divergence time & $-0 \cdot 334$ & $-25 \cdot 597$ & $0 \cdot 001$ \\
\hline & Geographical overlap & $0 \cdot 214$ & $1 \cdot 262$ & $0 \cdot 2$ \\
\hline \multirow[t]{2}{*}{$H_{0}$} & Divergence time & & & $0 \cdot 002$ \\
\hline & Geographical overlap & & & $0 \cdot 221$ \\
\hline
\end{tabular}

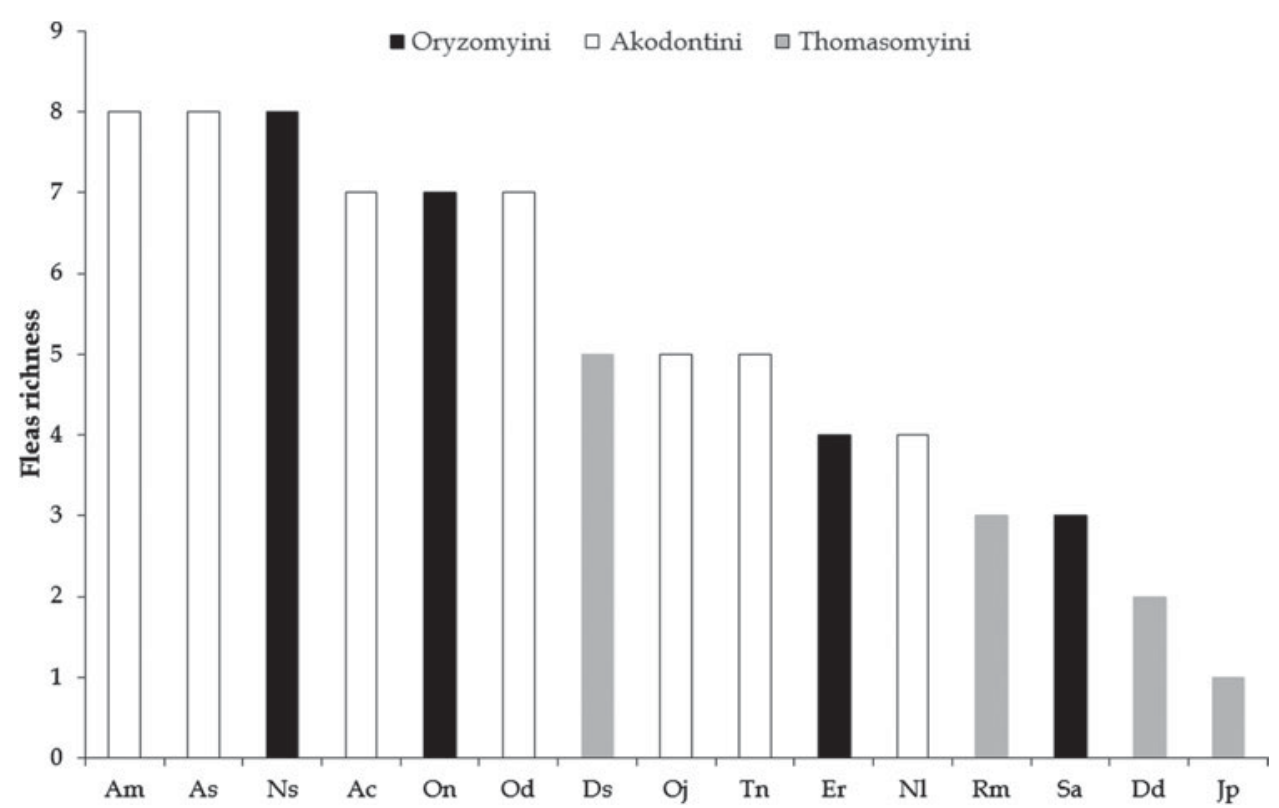

Fig. 3. The distribution of flea species richness on rodent hosts. (Hosts: Am, Akodon montensis; As, Akodon serrensis; Ns, Nectomys squamipes; Ac, Akodon cursor; On, Oligoryzomys nigripes; Od, Oxymycterus dasytrichus; Ds, Delomys sublineatus; Oj, Oxymycterus judex; Tn, Thaptomys nigrita; Er, Euryoryzomys russatus; Nl, Necromys lasiurus; Rm, Rhipidomys mastacalis; Sa, Sooretamys angouya; Dd, Delomys dorsalis and $\mathcal{F p}$, Fuliomys pictipes).

between $A$. montensis and $N$. squamipes $\left(\beta_{j}=0 \cdot 75\right)$. The mean Siphonaptera fauna size was $5 \cdot 13$ flea species per host species, with $23 \cdot 8 \%$ of the pairs of host species sharing at least 1 species of flea. The rodent species $A$. cursor and $A$. serrensis demonstrated the greatest similarity in parasitic flea species $\left(\beta_{j}=0 \cdot 87\right)$.

The mites and fleas showed high $S_{\mathrm{TD}}$ values (Mites: $n=17 ; \quad \bar{x}=2.64 \pm 0.22 ; \quad$ Fleas: $n=11$; $\bar{x}=2.37 \pm 0.29)$ (Fig. 1). The mite species Tur turki showed greater host specificity among mesostigmate species $\left(S_{\mathrm{TD}}=2.33\right)$ and was recorded exclusively on rodents belonging to the Akodontini tribe, whereas Gigantolaelaps matogrosensis, G. vitzthumi, and Laelaps castroi showed lower host specificity $\left(S_{\mathrm{TD}}=3\right)$ and were recorded on rodents belonging to the Akodontini and Oryzomyini tribes. In fleas, both Polygenis frustratus and P. lakoi showed the greatest host specificity $\left(S_{\mathrm{TD}}=2\right)$ with $P$. frustratus recorded on Akodontini rodents and $P$. lakoi recorded on Oryzomyini rodents, whereas $P$. roberti showed lower host specificity $\left(S_{\mathrm{TD}}=2 \cdot 75\right)$ and was recorded on rodents within the Akodontini, Oryzomyini, and Thomasomyini tribes. Comparison of the host specificity index between the mites and fleas showed that mesostigmate mites had lower host specificity than did the flea species (Mann-Whitney $U=157, P=0.003)$. The distribution of the species richness of mites (Fig. 2) and fleas (Fig. 3) shows that mites occur on both Akodontini and Oryzomyini rodents, whereas fleas occur mainly on Akodontini rodents.

The compositional similarities between the faunas of ectoparasites (Mesostigmata and Siphonaptera) by 


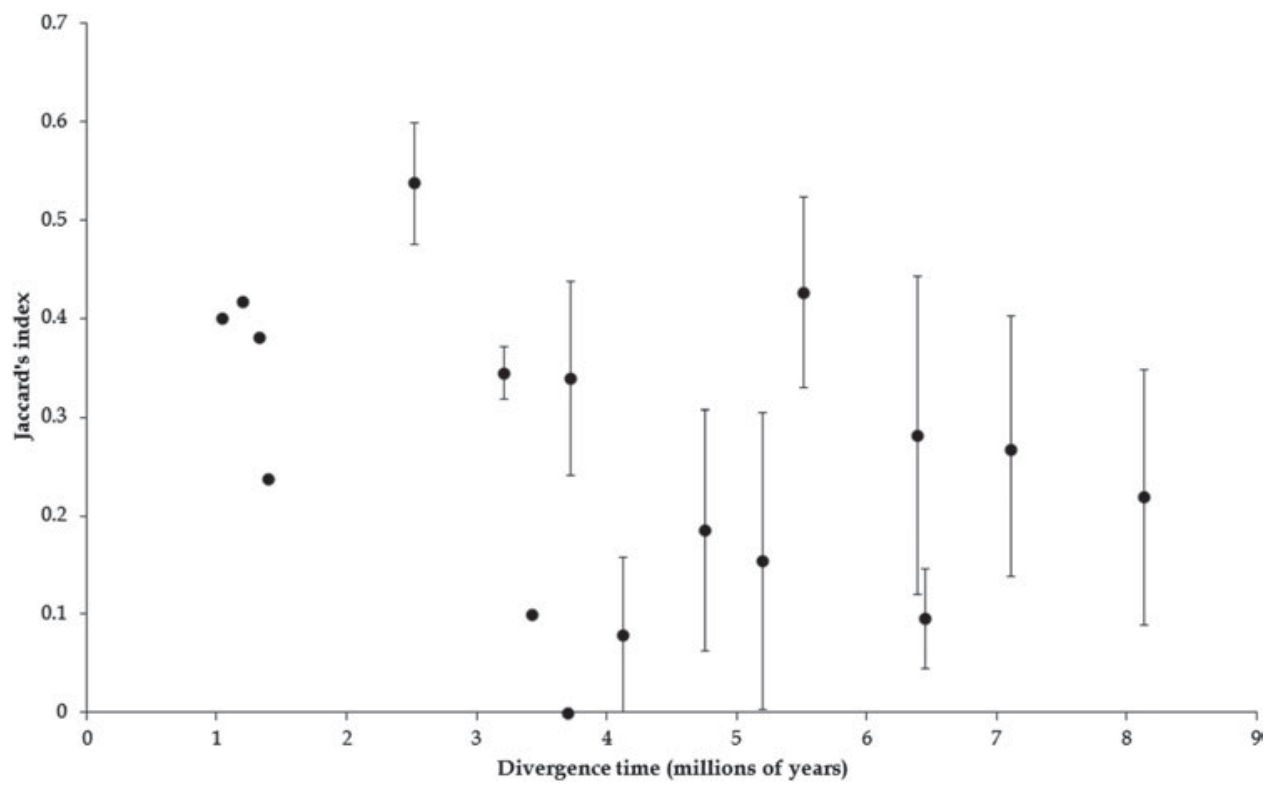

Fig. 4. Relationship between the divergence time and the proportion of total shared ectoparasite species (Mesostigmata and Siphonaptera). The filled circles correspond to the mean Jaccard's index $\left(\beta_{j}\right)$, whereas the bar represents the mean deviations.

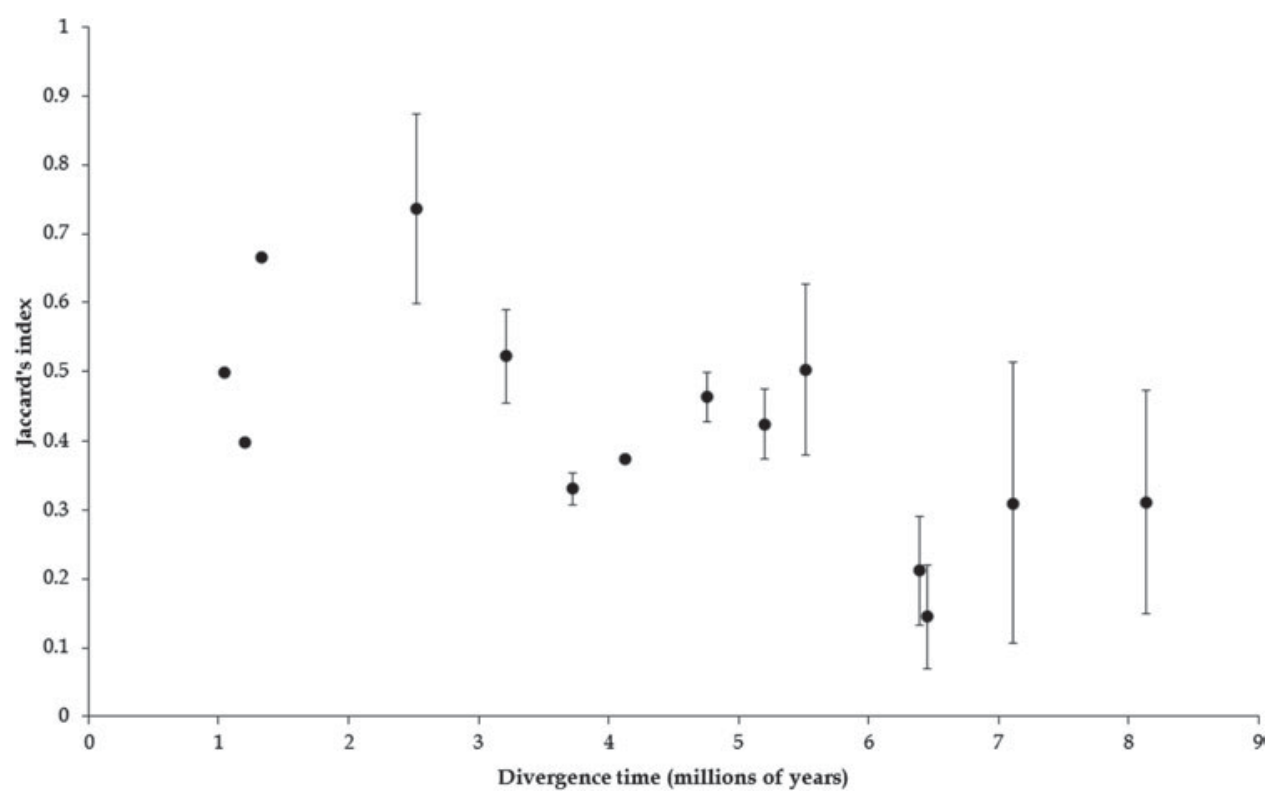

Fig. 5. Relationship between the divergence time and the proportion of shared Siphonaptera ectoparasite species. The filled circles correspond to the mean Jaccard's index $\left(\beta_{j}\right)$, whereas the bar represents the mean deviations.

the partial Mantel test indicated that the divergence time between the host species was the only significant predictor of whether an ectoparasite species was shared among the rodent hosts; host species that diverged more recently had ectoparasite faunas that were more similar than did species that had diverged earlier (Table 1, Fig. 4).

The partial Mantel test showed that both the divergence time and geographical overlap between the host species were not significant predictors of the compositional similarity of mesostigmate mite faunas (Table 1). Following the same pattern presented for the similarities among the faunas of the combined species of ectoparasites, the partial Mantel test showed that the divergence time between the host species was the only significant predictor of whether species of Siphonaptera were shared among the host species (Table 1, Fig. 5).

\section{DISCUSSION}

The host specificity index values in this study showed that fleas were more host-specific than were the 
mesostigmate mites. The reasons for this difference may be due to biological differences between these 2 groups of ectoparasites. Fleas are only blood-sucking ectoparasites during their adult stage, and although many fleas can feed on a variety of hosts, some flea species require a specific host to produce viable eggs and, thus, show a preference for a particular host (Bush et al. 2001). On the other hand, mesostigmate mites usually have more generalist food habits that range from predatory to parasitic blood feeding. In fact, in this study, all of the mesostigmate species belonged to the Laelapidae family, considered facultative nest and/or hair parasites, which are able to exploit a wide variety of hosts (Lindquist et al. 2009).

As predicted, the compositional similarities of flea faunas among sigmodontine rodents were influenced by the host divergence time, with species that had more recently diverged sharing more similar ectoparasite faunas than was observed between species that had diverged earlier. A similar influence of the host divergence time, or geographical distribution overlap, was not observed in the compositional similarities of the mite faunas. These results strongly resemble those from other studies on factors affecting similarities in ectoparasite faunas on rodent species. For example, Kransnov et al. (2010) studied rodentectoparasite systems of the Palaearctic region and showed that host phylogeny influenced the compositional similarities of the ectoparasite communities, but showed that environmental factors (e.g., vegetation and climatic conditions) also had a major influence. In the Neotropical region, Krasnov et al. (2012) showed that the phylogenetic dissimilarity of flea assemblages also was influenced by the phylogenetic dissimilarity of small mammal host assemblages. They suggested that fleas in the New World may simply have had less evolutionary time to switch between distantly related hosts than fleas in the Old World. Considering the evolutionary history of sigmodontine rodents, this also seems to be the case for flea species but not for mesostigmate mites in the Neotropical region.

The fact that neither host phylogeny nor geographical distribution influenced the composition of mesostigmate mite faunas indicates that another process may be responsible for the formation of parasite faunas, namely, the process of ecological fitting. 'Ecological fitting' describes a situation in which an organism interacts with its environment in a way suggestive of a shared evolutionary history when, in fact, the traits relevant to the interaction evolved elsewhere (or are due to an interaction with another organism) and in response to a different set of environmental conditions (Janzen, 1985). Thus, ecological fitting is a process whereby organisms colonize and persist in novel environments, use novel resources or form novel associations with other species (Agosta and Klemens, 2008). In this process, the colonization of novel hosts occurs because the parasite has pre-adaptations due to the new host sharing important characteristics with the current host or this adaptation may be the result of the parasite's phenotypic plasticity (Agosta et al. 2010). Thus, the similarities between the parasite faunas of host species may be determined largely by their morphological, physiological, and/or environmental similarities, which are unrelated to phylogeny and, in some cases, may be due to convergence.

In conclusion, this study found that phylogeny of the host species was the only factor that influenced compositional similarities among ectoparasite faunas. The study also indicated that flea acquisition, by the inheritance process, may be responsible for the formation of the Siphonaptera faunas on the rodent species studied. However, the lack of correlation between the similarity of mesostigmate mite faunas and both divergence time and geographical overlap suggested that other processes influenced the formation of these ectoparasite faunas. Ecological fitting may also underlie the formation of ectoparasite faunas and their similarities. Therefore, additional studies that account for other factors in the analysis can help to strengthen or refute the observed pattern of rodent-ectoparasite associations in Neotropical areas of high host species richness.

\section{ACKNOWLEDGEMENTS}

We thank Sergio Furtado dos Reis and José Alexandre Diniz Filho for data analysis suggestions. L. D. C. and F. R. F. received Doctoral Scholarships from Coordenação de Aperfeiçoamento de Pessoal de Nível Superior (CAPES).

\section{REFERENCES}

Agosta, S. J., Janz, N. and Brooks, D. R. (2010). How specialists can be generalists: resolving the "parasite paradox" and implications for emerging infectious disease. Zoologia 27, 151-162.

Agosta, S. J. and Klemens, J. A. (2008). Ecological fitting by phenotypically flexible genotypes: implications for species associations, community assembly and evolution. Ecology Letters 11, 1123-1134.

Antonovics, J., Hood, M. and Partain, J. (2002). The ecology and genetics of a host shift: Mycrobotryum as a model system. American Naturalist 160, S40-S53.

Brooks, D. R. (1988). Macroevolutionary comparisons of host and parasite phylogenies. Annual Review of Ecology and Systematics 19, 235-259.

Brooks, D. R., León-Règagnon, V., Mclennan, D. A. and Zelmer, D. (2006). Ecological fitting as a determinant of the community structure of platyhelminth parasites of anurans. Ecology 87 (Suppl.) S76-S85.

Bush, A. O., Fernandéz, J. C., Esch, G.W. and Seed, J. R. (2001). Parasitism: The Diversity and Ecology of Animal Parasites. Cambridge University Press, Cambridge, UK.

Davies, T.J. and Pedersen, A.B. (2008). Phylogeny and geography predict pathogen community similarity in wild primates and humans. Proceedings of the Royal Society of London, B 275, 1695-1701.

D’Elía, G. (2003). Phylogenetics of Sigmodontinae (Rodentia, Muroidea, Cricetidae), with special reference to the akodont group, and with additional comments on historical biogeography. Cladistics 19, 307-323.

Drummond, A. J., Ho, S. Y. M., Phillips, M. J. and Rambaut, A. (2006). Relaxed phylogenetics and dating with confidence. PLoS Biology 4, e88. Drummond, A. J. and Rambaut, A. (2007). BEAST: Bayesian evolutionary analysis by sampling trees. BMC Evolutionary Biology 7, 214. 
Hafner, M. S. and Page, R.D. M. (1995). Molecular phylogenies and host-parasite cospeciation: gophers and lice as a model system. Philosophical Transactions of the Royal Society, B 349, 77-83.

Hall, T. (2007). BioEdit v7.0.9: a User-Friendly Biological Sequence Alignment Editor and Analysis Program for Windows 95/98/N/2 K/XP/7. Available from: < http://www.mbio.ncsu.edu/bioedit/bioedit.html >

Hoberg, E.P. and Brooks, D. R. (2008). A macroevolutionary mosaic: episodic host-switching, geographical colonization and diversification in complex host-parasite systems. Fournal of Biogeography 35, 1533-1550.

Janzen, D. H. (1985) On ecological fitting. Oikos 45, 308-310.

Kennedy, C. R. and Bush, A. O. (1994). The relationship between pattern and scale in parasite communities: a stranger in strange land. Parasitology 109, 187-196.

Kimura, M. (1981). Estimation of evolutionary distances between homologous nucleotide sequences. Proceedings of the National Academy of Sciences, USA 78, 454-458.

Krasnov, B. R., Mouillot, D., Shenbrot, G. I., Khokhlova, I. S., Vinarski, M. V., Korallo-Vinarskaya, N.P. and Poulin, R. (2010) Similarity in ectoparasite faunas of Paleartic rodents as a function of hos phylogenetic, geographic or environmental distances: Which matters the most? International Fournal for Parasitology 40, 807-817.

Krasnov, B. R., Mouillot, D., Khokhlova, I. S., Shenbrot, G. I. and Poulin, R. (2012). Compositional and phylogenetic dissimilarity of hos communities drives dissimilarity of ectoparasite assemblages: geographical variation and scale-dependence. Parasitology 139, 338-347.

Krebs, C. J. (1999). Ecological Methodology, 2nd Edn. Benjamin Cummings, USA.

Koleff, P., Gaston, K. J. and Lennon, J. J. (2003). Measuring beta diversity for presence absence data. Fournal of Animal Ecology 72, 367-382 Lindquist, E. E., Krantz, G. W. and Walter, D. E. (2009). Order Mesostigmata. In A Manual of Acarology, 3rd Edn. (ed. Krantz, G. W. and Walter, D. E.), pp. 124-232. Texas Tech University Press, Lubbock, USA.

Manly, B. F. J. (1991). Randomization and Monte Carlo Methods in Biology. Chapman and Hall, London, UK.

Muñoz, G., Grutter, A.S. and Cribb, T.H. (2006). Endoparasite communities of five fish species (Labridae: Cheilininae) from Lizard Island: how important is the ecology and phylogeny of the hosts? Parasitology 132, 363-374.

Oksanen, J., Blanchet, F. G., Kindt, R., Legendre, P., O'Hara, R. B. Simpson, G. L., Solymos, P., Stevens, M. H. H. and Wagner, H. (2011) Vegan: Community Ecology Package. R package version 1.17-6. Available from: < http://CRAN.R-project.org/package=vegan $>$

Pardiñas, U. F. J., D'Elía, G. and Ortiz, P. E. (2002). Sigmodontino fósiles (Rodentia, Muroidea, Sigmodontinae) de América del Sur: Estado actual de su conocimiento y prospectiva. Fournal of Neotropical Mammalogy 9, 209-252.
Paterson, A. M., Palma, R. L. and Gray, R. D. (1999). How frequently do avian lice miss the boat? Implications for coevolutionary studies. Systematic Biology 48, 214-223.

Patterson, B. D., Ceballos, G., Sechrest, W., Tognelli, M. F. Brooks, T., Luna, L., Ortega, P., Salazar, I. and Young, B. E. (2007) Digital Distribution Maps of the Mammals of the Western Hemisphere, version 3.0. NatureServe, Virginia, USA. Available from: < http://www.nature serve.org $>$.

Posada, D. (2008). jModelTest: Phylogenetic Model Averaging. Molecular Biology and Evolution 25, 1253-1256.

Poulin, R. (2007). Evolutionary Ecology of Parasites, 2nd Edn. Princeton University Press, Princeton, NJ, USA.

Poulin, R. (2010). Decay of similarity with host phylogenetic distance in parasite faunas. Parasitology 137, 733-741

Poulin, R. and Mouillot, D. (2003). Parasite specialization from a phylogenetic perspective: a new index of host specificity. Parasitology 126, 473-480.

Rangel, T.F., Diniz-Filho, J. A. F. and Bini, L. M. (2010). SAM: a comprehensive application for Spatial Analysis in Macroecology. Ecography 33, 46-50.

R Development Core Team. (2010). R: A Language and Environment for Statistical Computing. R Foundation for Statistical Computing, Vienna, Austria. Available from: <http://www.r-project.org >

Reig, O. A. (1987). An assessment of the systematics and evolution of the Akodontini, with the description of new fossil species of Akodon (Cricetidae: Sigmodontinae). Fieldiana Zoology 39, 347-399.

Rosenberg, M.S. and Anderson, C. D. (2011). PASSaGE: Pattern Analysis, Spatial Statistics and Geographic Exegesis. Version 2. Methods in Ecology and Evolution 2, 229-232.

Smith, M. F. and Patton, J. L. (1999). Phylogenetic relationships and the radiation of sigmodontine rodents in South America: evidence from cytochrome b. Fournal of Mammalian Evolution 6, 89-128.

Smith, V.S., Light, J. E. and Durden, L. A. (2008). Rodent louse diversity, phylogeny, and cospeciation in the Manu Biosphere Reserve, Peru. Biological Fournal of the Linnean Society 95, 598-610.

Smouse, P. E., Long, J. C. and Sokal, R. R. (1986). Multiple regression and correlation extensions of the Mantel test of matrix correspondence. Systematic Zoology 35, 627-632.

Spradling, T. A., Hafner, M. S. and Demastes, J. W. (2001). Differences in rate of cytochrome- $b$ evolution among species of rodents. Fournal of Mammalogy 82, 65-80.

Steppan, S. J., Adkins, R. M. and Anderson, J. (2004). Phylogeny and divergence-date estimates of rapid radiations in muroid rodents based on multiple nuclear genes. Systematic Biology 53, 533-553.

Weksler, M. (2003). Phylogeny of Neotropical oryzomyine rodents (Muridae: Sigmodontinae) based on the nuclear IRBP exon. Molecula Phylogenetics and Evolution 29, 331-349. 